\title{
Propofol-Induced Masseter Muscle Spasm in a Woman with a Major Depressive Disorder
}

\author{
Mohammad Saeidi, ${ }^{1}$ Rosa Alikhani, ${ }^{2}$ Ahmad Hormati, ${ }^{3}$ Seyed Mehdi Sabouri, ${ }^{1}$ and Reza Aminnejad ${ }^{1,{ }^{*}}$ \\ ${ }^{1}$ Department of Anesthesiology and Critical Care, Qom University of Medical Sciences, Qom, Iran \\ ${ }^{2}$ Department of Psychiatry, University of Social Welfare and Rehabilitation Sciences, Tehran, Iran \\ ${ }^{3}$ Gastroenterology and Hepatology Disease Research Center, Qom University of Medical Sciences, Qom, Iran \\ "Corresponding author: Reza Aminnejad, Department of Anesthesiology and Critical Care, Qom University of Medical Sciences, Qom, Iran. E-mail: r.aminnejad@yahoo.com \\ Received 2018 May 13; Revised 2018 June 10; Accepted 2018 June 17.
}

\begin{abstract}
Masseter muscle rigidity is a known complication of drugs such as succinylcholine and volatile agents. However, muscle rigidity is an uncommon complication of propofol. We report the case of a 56-year-old ASA-PS class I woman refereeing for colonoscopy due to chronic constipation under deep intravenous sedation. She suffered masseter spasm after the injection of propofol. Masseter spasm should not be considered limited to special drug groups. In any case of difficult mouth opening, masseter spasm should be kept in mind and deepening of anesthesia or complete blockade of neuromuscular junction should be considered by the use of non-depolarizing muscle relaxants.
\end{abstract}

Keywords: Anesthesia, Major Depressive Disorder, Masseter Spasm, Propofol

\section{Introduction}

Masseter muscle rigidity is a known complication of drugs such as succinylcholine and volatile agents, especially in patients with underlying muscular diseases such as myotonia and malignant hyperthermia ( $\mathrm{MH})$. However, this complication can be seen following the administration of non-depolarizing muscle relaxants such as rocuronium, vecuronium, and pancuronium (1). In this case report, we present an otherwise healthy case who suffered from masseter spasm while anesthesia did not involve common guilty drugs.

\section{Case Presentation}

We report the case of a 56-year-old ASA-PS class I woman refereeing for colonoscopy due to chronic constipation under general anesthesia. She had a history of uneventful general anesthesia for appendectomy 2 years ago. We did not have any record denoting the drugs used for her previous anesthesia. In addition, she had a history of depression treated by Sertraline (total daily dose of $200 \mathrm{mg}$ ) up to 6 months ago. In the physical examination, there was no abnormal finding and the airway examination did not show any difficulty in ventilation or intubation. All laboratory tests were acceptable. After usual preparation and pre- medication with $2 \mathrm{mg}$ of midazolam and $100 \mathrm{mcg}$ of fentanyl, auto-co-induction was performed by $30 \mathrm{mg}$ propofol. 3 minutes later, $70 \mathrm{mg}$ ( $1 \mathrm{mg} / \mathrm{Kg}$ ) propofol was injected intravenously for the induction of general anesthesia. Suddenly, the patient fell into an uncontrollable agitated state that forced us to inject another dose of propofol $(70 \mathrm{mg})$ for deepening the anesthesia and use the oropharyngeal airway for maintaining mouth opening. A few seconds later, the patient was calm and became apneic, mandating bag ventilation via facemask, but difficult mouth opening and mask ventilation. At this time, we decided to terminate the procedure and awake the patient. Fortunately, after a few minutes, she began to breathe spontaneously and after a while, she regained her consciousness gradually. In all the time of anesthesia, hemodynamic changes and airway pressure were in an acceptable range. We did not detect either hyperthermia or increased end-tidal carbon dioxide concentration (in Capnography). Rigidity in other muscles was not detected either during anesthesia or in the postanesthesia care unit. When the patient gained her complete consciousness, she complained of severe pain in jaws and neck and she described the jaws pain as a reason for agitation. After an uneventful recovery phase for an hour, the patient was transferred to the ward while she was treated by apotel and pethidine for the jaws and neck pain. A few days later, she had no pain and mouth opening was in the 
normal range again as before anesthesia. When we asked about the same experience in patient's family, neither she nor her accompanying family could remember any relevant event. The patient did not come back to our center for completing the gastrointestinal investigation for her underlying discomfort anymore.

\section{Discussion}

Muscle rigidity is an uncommon complication of propofol and this hypnotic drug can be used to reduce muscle rigidity caused by other drugs such as succinylcholine in usual practice $(2,3)$. Muscle rigidity can be part of the serotonin syndrome caused by excessive amounts of serotonin in the central nervous system due to SSRI (selective serotonin reuptake inhibitor) overdose (4). Our case was a major depressive disordered woman treated for a long time with sertraline (total daily dose of $200 \mathrm{mg}$ ) up to 6 months before admission and she stopped her pharmacologic therapy arbitrarily. She was mentally in a good condition on the day of the procedure. Preoperative anxiety can be a predisposing factor to the occurrence of masseter muscle spasm maybe due to inducing involuntary clenching of jaws (5). In this case, masseter muscle spasm occurred immediately after an induction dose of propofol. Our patient had an easy airway predicted in pre-procedural airway examinations. She could open her mouth for more than $4 \mathrm{~cm}$ with normal mobility in the temporomandibular joint, but after administering $100 \mathrm{mg}$ of propofol, difficult mouth opening prevented us to continue bag-valvemask ventilation. At this time, we decided to maintain mouth opening with the use of oropharyngeal airway. Fortunately, the chest wall rigidity was not detected and ventilation continued without extreme difficulty after apply- ing an oropharyngeal airway. In such cases, thinking of the probability of $\mathrm{MH}$ is rational because it is potentially fatal. For this purpose, temperature monitoring and looking for rigidity and spasm in other muscle groups are prudent (6).

\subsection{Conclusion}

Masseter spasm following the induction of anesthesia should not be considered limited to special drug groups. In any case of difficult mouth opening, masseter spasm should be kept in mind and deepening of anesthesia or complete blockade of neuromuscular junction should be considered by the use of non-depolarizing muscle relaxants.

\section{References}

1. Raut MS, Maheshwari A, Jyoti A, Joshi S. 'Jaws of steel' after rocuronium. Indian J Anaesth. 2016;60(2):141-2. doi: 10.4103/0019 5049.176275. [PubMed: 27013757]. [PubMed Central: PMC4787129].

2. Bleeg RC, Rasmussen BS, Lambert PH. [Propofol-induced trismus, causing an unanticipated difficult intubation due to masseter spasm]. Ugeskr Laeger. 2014;176(30). [PubMed: 25292243].

3. Elsharydah A, Kaminski AC. Propofol Reduces Succinylcholineinduced Muscle Rigidity in a Patient with Paramyotonia Congenita. Anesth Essays Res. 2017;11(1):273-4. doi: 10.4103/0259-1162.200242. [PubMed: 28298805]. [PubMed Central: PMC5341655].

4. Fitzgerald KT, Bronstein AC. Selective serotonin reuptake inhibitor exposure. Top Companion Anim Med. 2013;28(1):13-7. doi: 10.1053/j.tcam.2013.03.003. [PubMed: 23796482].

5. Landman JJ, Verhamme Y, Tielens LK, Scheffer GJ. Persistent masseter spasm during anaesthesia. Anaesthesia. 2011;66(12):1180. doi: 10.1111/j.1365-2044.2011.06950.x. [PubMed: 22070596].

6. In J, Ahn EJ, Lee DK, Kang H. Incidence of malignant hyperthermia in patients undergoing general anesthesia: Protocol for a systematic review and meta-analysis. Medicine (Baltimore). 2017;96(49). e9115. doi: 10.1097/MD.0000000000009115. [PubMed: 29245345]. [PubMed Central: PMC5728960]. 